\title{
Fístula colovesical secundaria a enfermedad diverticular: cirugía laparoscópica electiva*
}

\author{
Drs. OCTAVIO A. CASTILLO C. ${ }^{1,2,3}$, ARQUÍMEDES RODRÍGUEZ-CARLIN, \\ GONZALO CAMPAÑA V. ${ }^{4}$, ALBERTO PÉREZ C. ${ }^{4}$ \\ 1 Unidad de Urología, Clínica Indisa. \\ 2 Facultad de Medicina, Universidad Andrés Bello. \\ 3 Facultad de Medicina, Universidad de Chile. \\ 4 Unidad de Cirugía, Clínica Indisa. \\ Santiago, Chile.
}

\begin{abstract}
\section{Colovesical fistula: laparoscopic surgery}

Aim: Colovesical fistula is a severe complication associated to neoplastic or inflammatory colon disease. Most common procedure is open surgery. We report a case of colovesical fistula secondary to diverticular disease treated with a laparoscopic approach. Methods: A 64-year-old man was seen with a known colonic diverticular disease, type 2 diabetes mellitus and recurrent urinary tract infection. He complained of lower abdominal pain, dysuria, and pneumaturia. A CT scan revealed a sigmoid diverticular perforation into the bladder (colovesical fistula). A left hemicolectomy with partial cystectomy was performed by laparoscopy means. Results: There was no morbidity related to the surgical procedure, and the final pathology confirmed a colonic diverticular disease with bladder compromise. The patient is asymptomatic at 24 months of follow up. Conclusion: The laparoscopic approach is a feasible alternative for the treatment of colovesical fistula with low morbidity.
\end{abstract}

Key words: Colovesical fistulae, diverticular disease, bowel inflamatory diseases, colonic surgery, laparoscopic surgery.

\section{Resumen}

Objetivos: La fístula colovesical es una complicación relacionada con procesos inflamatorios y neoplásicos del colon. El manejo tradicional de esta patología es quirúrgico bajo técnica abierta. Presentamos un caso de fístula colovesical secundaria a enfermedad diverticular con tratamiento quirúrgico laparoscópico. Materiales y Métodos: Paciente masculino de 64 años de edad, portador de enfermedad diverticular de larga data con antecedente de diabetes mellitus tipo 2 e infecciones urinarias a repetición, quien consulta por presentar dolor en hipogastrio, disuria y neumaturia. Se realiza tomografía computada que revela perforación diverticular de colon sigmoides a vejiga (fístula colovesical). Se realizó hemicolectomía izquierda más cistectomía parcial, con resección del trayecto fistuloso, y cierre vesical y anastomosis colónica laparos-

*Recibido el 15 de junio de 2011 y aceptado para publicación el 22 de enero de 2012.

Correspondencia: Dr. Octavio A. Castillo C.

Apoquindo 3990, Of. 809, Santiago, Chile. C.P. 7550112. Fax (56-2) 2282425

octavio.castillo@indisa.cl 
cópica. Resultados: No hubo complicaciones intra ni postoperatorias. El estudio anatomopatológico de la pieza operatoria demostró enfermedad diverticular. El paciente se encuentra asintomático a los 24 meses de seguimiento. Conclusión: El abordaje laparoscópico es una alternativa factible para el tratamiento de fístula colovesical, con baja morbilidad.

Palabras clave: Fístula colovesical, enfermedad diverticular, enfermedad inflamatoria, cirugía de colon, cirugía laparoscópica.

\section{Introducción}

Las fístulas colovesicales son poco frecuentes y representan un desafío en su diagnóstico y tratamiento. La etiología más frecuente es la enfermedad diverticular complicada (66\% de los casos) y el cáncer de colon (alrededor del 20\% de los casos). Causas menos frecuentes son la enfermedad de Crohn, daño actínico y lesiones iatrogénicas secundarias a cirugía de recto y vejiga ${ }^{1}$. Aunque el objetivo del tratamiento es garantizar la resolución de la fístula con la restitución del tránsito intestinal, las opciones van desde una derivación con colostomía transitoria hasta cirugía en un tiempo. Existen en la literatura quirúrgica escasas publicaciones sobre el tratamiento laparoscópico de la fístula colovesical, procedimiento técnicamente difícil y con una alta tasa de conversión².

Presentamos un caso de fístula colovesical secundaria a enfermedad diverticular, la cual se manejó exitosamente con tratamiento quirúrgico laparoscópico en un tiempo.

\section{Caso clínico}

Paciente de 64 años de edad, sexo masculino, con el antecedente de diabetes mellitus tipo 2 y de enfermedad diverticular colónica conocida de larga data, con el antecedente de infecciones urinarias

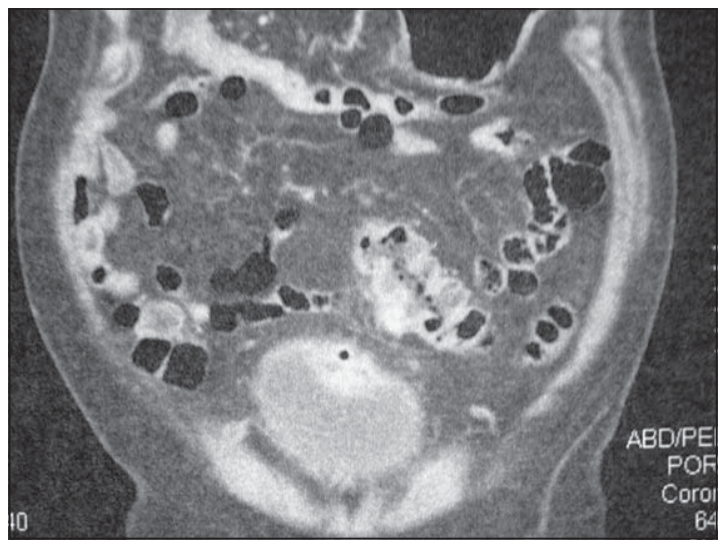

Figura 1. Tomografía computada en corte transversal que muestra proceso inflamatorio de la cúpula y una burbuja de aire en su interior. recurrentes. Consulta por presentar dolor en hipogastrio y fosa ilíaca izquierda, disuria y neumaturia intermitentes. El cultivo de orina mostraba el desarrollo de Proteus spp más de 100.000 UFC. Ante el diagnóstico clínico de Enfermedad diverticular complicada con fístula colo-vesical se realiza Tomografía computada de abdomen y pelvis con contraste (TC) la cual confirma la presencia de una perforación diverticular de colon sigmoides a vejiga (Figuras $1 \mathrm{y}$ 2), sin evidencias de un proceso inflamatorio agudo. Se plantea al paciente la necesidad de una cirugía electiva, con resección colónica y de la fístula colovesical por vía laparoscópica, enfatizando la eventual necesidad de conversión a cirugía abierta.

\section{Técnica quirúrgica}

Se realizó preparación intestinal con Fleet Fosfosada oral ${ }^{\circledR}$ y se administró Cefotaxima y Metronidazol intravenoso durante la inducción anestésica.

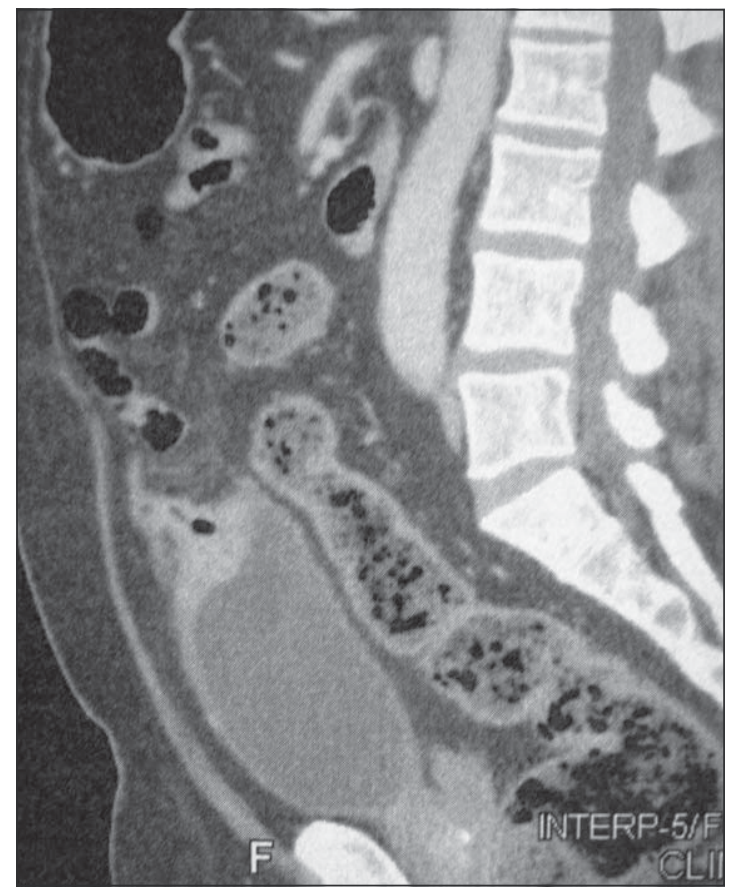

Figura 2. Corte sagital que muestra la proximidad la vencindad de colon a la cúpula vesical y el proceso inflamatorio asociado. 
Se coloca el paciente en decúbito dorsal con inclinación de $20^{\circ}$ en posición de Trendelenburg (Posición de Lloyd Davis modificada). Se realiza neumoperitoneo con aguja de Veress a $15 \mathrm{mmHg}$. La posición de los trocares es la siguiente: 1 trocar de $10 \mathrm{~mm}$ supraumbilical para la cámara, 2 trocares de $10 \mathrm{~mm}$ paraumbilical y fosa ilíaca derecha y un trocar de $5 \mathrm{~mm}$ en fosa ilíaca izquierda (Figura 3). La inspección abdominal revela un proceso inflamatorio moderado con múltiples adherencias entre colon sigmoides y epiplón, apreciándose la adherencia del colon sigmoides a la cúpula vesical. Luego de liberadas las adherencias se procede a liberar el colon sigmoides y la vejiga, englobando la zona inflamatoria que incluye la fístula. Luego se procede a resección subtotal de la vejiga sobre tejido sano y sutura intracorpórea vesical con un plano mucoso de Vycril 3-0 y un segundo plano seromuscular de Monocryl 2-0. Se inicia la disección de la corredera parieto-cólica izquierda identificando el uréter izquierdo. Luego por medial se liga la arteria mesentérica inferior con Weck clip ${ }^{\circledR}$. Se diseca la unión recto sigmoidea y se secciona con una engrapadora con carga intestinal de 45 mm (Endo - GIA, Ethicon Endo-Surgery, Cincinnati, Ohio, EE.UU). Se realiza una incisión de Pfannenstiel de $6 \mathrm{~cm}$ por donde se exterioriza el colon sigmoides que se secciona a proximal y se introduce el cabezal de la engrapadora circular no 29 (CEEA; Ethicon Endosurgery, Cincinnati, Ohio, EE.UU), y se fija mediante una jareta de Polipropileno 2-0. Se reintroduce el colon proximal con el cabezal de la engrapadora, y se rehace el neumoperitoneo. Se introduce la engrapadora circular por vía anal para realizar la anastomosis colorrectal intracorpórea. Se realiza una prueba de hermeticidad demostrando una anastomosis colorrectal a 17 $\mathrm{cm}$ con indemnidad de la anastomosis y del colon proximal. La pieza quirúrgica muestra los segmentos colónico y vesical, y el trayecto fistuloso (Figura 4).

\section{Resultados}

El tiempo operatorio fue de 150 minutos. El sangrado estimado fue $50 \mathrm{ml}$ y no hubo complicaciones perioperatorias. Se reinició alimentación oral al $3^{\circ}$ día de postoperatorio indicándose el alta al $5^{\circ}$ día de postoperatorio. Actualmente, el paciente se encuentra asintomático a los 24 meses de seguimiento.

\section{Discusión}

La fístula enterovesical es una patología poco frecuente, lo que plantea un desafío para su diagnóstico y tratamiento. La fístula colovesical (FCV) es la más

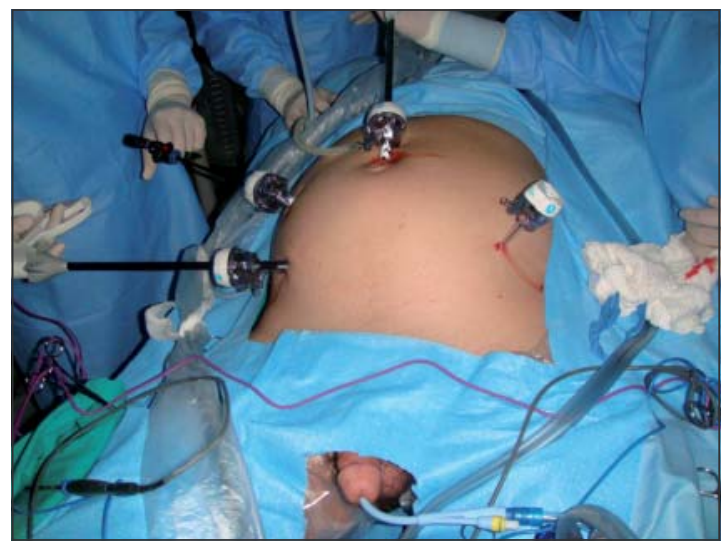

Figura 3. Posición de los puertos de trabajo.

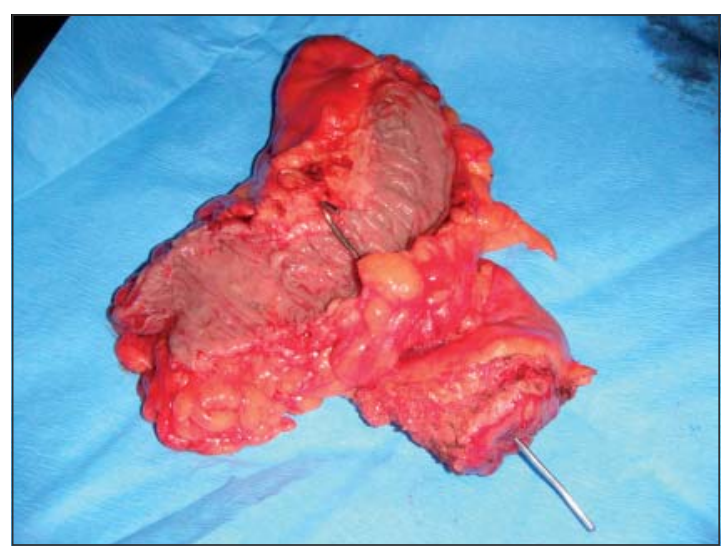

Figura 4. Pieza quirúrgica, colon sigmoides y cúpula vesical, con una sonda metálica que define el trayecto fistuloso.

frecuente (70\%), seguida por la ileovesical (16\%) y rectovesical $(11 \%)^{3}$. Es más común en el sexo masculino en una proporción de 3:1 y las décadas de mayor prevalencia son la sexta y la séptima ${ }^{4}$.

La causa más frecuente de fístula colovesical es la enfermedad diverticular del colon sigmoides (66-75\% de los casos) ${ }^{1,5}$. El mecanismo subyacente es la extensión directa de un divertículo roto o la erosión de un absceso peri-diverticular hacia la vejiga. También se ha descrito como consecuencia de cáncer de colon, radioterapia y enfermedad de Crohn. La mayoría de los pacientes (> 90\%) con fístula colovesical suelen presentar infecciones urinarias a repetición y disuria ${ }^{5}$. La neumaturia está presente en un $71-90 \%$ de los casos y la fecaluria en un 51-76\%, ambos signos considerados signos patognomónicos de $\mathrm{FCV}^{5,6}$.

Estudios previos han demostrado que hasta el $75 \%$ de los pacientes con fístula colovesical no 
tratados quirúrgicamente, morirán a causa de complicaciones sépticas. Garcea y cols, manejaron 18 pacientes con tratamiento médico y 2 (11\%) de los pacientes de este grupo murieron, específicamente de sepsis urinaria ${ }^{6}$.

Bannura y cols, observaron una incidencia de fístula colónica de 19,1\% en 141 pacientes intervenidos electivamente por enfermedad diverticular, de las cuales el 72\% fueron colovesicales. Encontraron que el $83 \%$ de los pacientes con FCV presentaron neumaturia y el 56\% fecaluria. La tomografía computada fue el estudio más relevante para demostrar la presencia de la fístula colovesical. El tratamiento empleado por ellos en el $94 \%$ de los pacientes fue sigmoidectomía con anastomosis colorrectal por técnica abierta más rafia vesical. El promedio de hospitalización fue de 12,8 días y no hubo reoperaciones ni mortalidad operatoria ${ }^{7}$.

Bartus y cols, demostraron que la colectomía laparoscópica electiva es una opción de tratamiento factible en la enfermedad diverticular complicada con fístula. Ellos compararon un grupo de pacientes con enfermedad diverticular sintomática no complicada y otro grupo con enfermedad diverticular complicada con fístula colovesical. A todos los pacientes se les realizó colectomía laparoscópica electiva. No hubo fuga en la anastomosis ni episodios de sangrado que requirieran de reoperación en el grupo con fístula. No hubo diferencia estadísticamente significativa en el período de estancia hospitalaria y tiempo operatorio entre ambos grupos ${ }^{8}$.

En Chile hay reportes de cirugía laparoscópica de colon con resultados similares a la cirugía abierta. Zárate y cols, compararon los resultados y complicaciones inmediatas al realizar sigmoidectomía laparoscópica versus abierta en pacientes con cáncer de colon sigmoides, obteniendo resultados quirúrgicos similares ${ }^{9}$. Recientemente se realizó la primera proctocolectomía laparoscópica con reservorio íleo-anal, sin ileostomía de protección, practicada a un paciente con poliposis adenomatosa familiar con buenos resultados quirúrgicos y funcionales ${ }^{10}$.
A pesar de que presentamos sólo un caso clínico aislado de fístula colo-vesical tratada en un tiempo por cirugía laparoscópica, consideramos que esta técnica debe ser una alternativa planteable en la resolución quirúrgica de esta patología.

\section{Referencias}

1. Dorairajan LN, Hemal AK. Lower urinary tract fistula: the minimally invasive approach. Curr Opin Urol. 2009;19:556-62.

2. Pokala N, Delaney CP, Brady KM, Senagore AJ. Elective laparoscopic surgery for benign internal enteric fistulas: a review of 43 cases. Surg Endosc. 2005;19:222-5.

3. Pontari MA, McMillen MA, Garvey RH, Ballantyne GH. Diagnosis and treatment of enterovesical fistulae. Am Surg. 1992;58:258-63.

4. Scozzari G, Arezzo A, Morino M. Enterovesical fistulas: diagnosis and management. Tech Coloproctol. 2010;14:293-300.

5. Garcea G, Majid I, Sutton CD, Pattenden CJ, Thomas WM. Diagnosis and management of colovesical fistulae; six-year experience of 90 consecutive cases. Colorectal Dis. 2006;8:347-52.

6. Melchior S, Cudovic D, Jones J, Thomas C, Gillitzer $\mathrm{R}$, Thüroff J. Diagnosis and surgical management of colovesical fistulas due to sigmoid diverticulitis. J Urol. 2009;182:978-82.

7. Bannura G, Barrera A, Cumsille M, Melo C, Soto D, Contreras J. Fístula colovesical de origen diverticular. Rev Chil Cir. 2010;62:49-54.

8. Bartus CM, Lipof T, Sarwar CM, Vignati PV, Johnson KH, Sardella WV, Cohen JL. Colovesical fistula: not a contraindication to elective laparoscopic colectomy. Dis Colon Rectum 2005;48:233-6.

9. Zárate A, López-Köstner F, Loureriro C, Pinedo G, Molina M, Kronberg U, y cols. Resultados y eventos adversos de la sigmoidectomía por cáncer: laparoscopia versus laparotomía. Rev Chil Cir. 2008;60:29-34.

10. López-Köstner F, Zárate A. Proctocolectomía restauradora con reservorio íleoanal laparoscópica sin ileostomía de protección. Rev Chil Cir. 2008;60:63-6. 